\title{
Dynamic Analysis of Wind Turbine Blades Using Radial Basis Functions
}

\author{
Ming-Hung Hsu \\ Department of Electrical Engineering, National Penghu University of Science and Technology, Penghu 880, Taiwan \\ Correspondence should be addressed to Ming-Hung Hsu, hsu.mh@msa.hinet.net
}

Received 30 October 2010; Revised 11 April 2011; Accepted 11 April 2011

Academic Editor: K. M. Liew

Copyright () 2011 Ming-Hung Hsu. This is an open access article distributed under the Creative Commons Attribution License, which permits unrestricted use, distribution, and reproduction in any medium, provided the original work is properly cited.

Wind turbine blades play important roles in wind energy generation. The dynamic problems associated with wind turbine blades are formulated using radial basis functions. The radial basis function procedure is used to transform partial differential equations, which represent the dynamic behavior of wind turbine blades, into a discrete eigenvalue problem. Numerical results demonstrate that rotational speed significantly impacts the first frequency of a wind turbine blade. Moreover, the pitch angle does not markedly affect wind turbine blade frequencies. This work examines the radial basis functions for dynamic problems of wind turbine blade.

\section{Introduction}

Wind energy will likely play an important role as a future energy source in countries worldwide. Energy is essential to economic growth. In response to environmental concerns, the amount of energy generated using renewable resources is increasing [1-3]. Herbert et al. [2] reviewed assessment models for wind resources, site selection models, and aerodynamic models, including the wake effect model. Varol et al. [3] demonstrated the positive effect of aerofoils surrounding wind blades. Their notion of surrounding aerofoils resembles that of steering blades in a water turbine. Spee et al. [4] created a novel control strategy for a brushless doubly fed machine applied to variable-speed wind-power generation systems. Vitale and Rossi [5] designed lowpower, horizontal-axis wind turbine blades, using an iterative algorithm. With their software, one can determine the optimum blade shape for a wind turbine to satisfy the energy requirements of an electrical system with optimum rotor efficiency. Song and Dhinakaran [6] developed a nonlinear wind turbine control method. Storti and Aboelnaga [7] analyzed transverse deflections of a straight tapered symmetrical beam attached to a rotating hub as a model for bending vibration of blades in turbomachinery. The natural frequencies of a single tapered and pretwisted turboblade were calculated by Rao [8, 9], Abrate [10], Hodges et al. [11], and Dawson and Carneige $[12,13]$ using the Rayleigh-Ritz scheme. Gupta and Rao [14] applied the finite element method to identify the frequencies of natural vibration of doubly tapered and twisted beams. Swaminathan and Rao [15] described the vibrations of a rotating pretwisted and tapered blade. Subrahmanyam et al. $[16,17]$ derived the natural frequencies and mode shapes of a uniform pretwisted cantilever blade using the Reissner approach. Chen and Keer [18] examined the transverse vibrations of a rotating pretwisted Timoshenko beam under axial loading. Storti and Aboelnaga [19] examined transverse deflections of a straight tapered symmetrical beam attached to a rotating hub as a model for the bending vibration of blades. Wagner [20] derived the forced vibration response of subsystems with different natural frequencies and damping attached to a foundation with a finite stiffness and mass. Griffin and Sinha [21-23] determined the dynamic responses of frictionally damped turbine blades. Determining the dynamic features of wind turbine blades is of significant importance. Radial basis functions are important elements of approaches generally called meshless methods. In this study, dynamic problems associated with wind turbine blades are formulated using radial basis functions.

\section{Radial Basis Function}

A radial basis function is a real-value function whose value depends on distance from an origin. Kansa $[24,25]$ 
investigated a given function or partial derivatives of a function with respect to a coordinate direction expressed as a linear weighted sum of all functional values at all mesh points along the direction initiated based on the concept of the radial basis function. In their algorithm, node distribution was entirely unstructured. Wang and Liu [26] developed a point interpolation meshless method based on radial basis functions that incorporated the Galerkin weak form for solving partial differential equations. Elfelsoufi [27] investigated buckling, flutter, and vibration of beams using radial basis functions. Hon et al. [28] utilized radial basis functions for function fitting and solving partial differential equations using global nodes and collocation procedures. Liu et al. [29] constructed shape functions with the delta function property using radial and polynomial basis functions. Devi and Pepper [30] generated a simplified radial basis function approach for calculating coupled heat transfer of a fluid flow using a local pressure correction scheme. In their study, shape functions were constructed using radial basis functions. Vrankar et al. [31] applied a relatively new approach for modeling radionuclide migration through the geosphere using a radial basis function scheme. Qiao and Ernst [32] applied a nonlinear approach for constructing color conversions based on radial basis functions. Their experimental results demonstrated that color conversion can be achieved effectively using the radial basis function approach when constructing nonlinear data maps. A radial basis function can be expressed as follows $[33,34]$ :

$$
B_{i}(r)=\sqrt{\left(r-r_{i}\right)^{2}+c^{2}},
$$

where $c$ is a shape parameter. The radial basis function is generally utilized to develop functional approximations in the following forms $[33,34]$ :

$$
\begin{aligned}
u_{k}(r, t) & =\sum_{i=1}^{N} a_{k i}(t) B_{i}(r), \quad \text { for } k=1,2,3, \\
v_{k}(r, t) & =\sum_{i=1}^{N} \bar{a}_{k i}(t) B_{i}(r), \quad \text { for } k=1,2,3, \\
U_{k}(r) & =\sum_{i=1}^{N} b_{k i} B_{i}(r), \quad \text { for } k=1,2,3, \\
V_{k}(r) & =\sum_{i=1}^{N} \bar{b}_{k i} B_{i}(r), \quad \text { for } k=1,2,3,
\end{aligned}
$$

where $a_{k i}, \bar{a}_{k i}, b_{k i}$, and $\bar{b}_{k i}$ are coefficients to be determined. Blade deflection $u_{k}(r, t)$ is the sum of $N$ radial basis functions, each associated with a different center $r_{i}$. Blade deflection $v_{k}(r, t)$ denotes the sum of $N$ radial basis functions, each associated with a different center $r_{i}$. Blade deflection $U_{k}(r)$ is the sum of $N$ radial basis functions, each associated with a different center $r_{i}$. Blade deflection $V_{k}(r)$ denotes the sum of $N$ radial basis functions, each associated with a different center $r_{i}$. The domain contains $N$ collocation points.

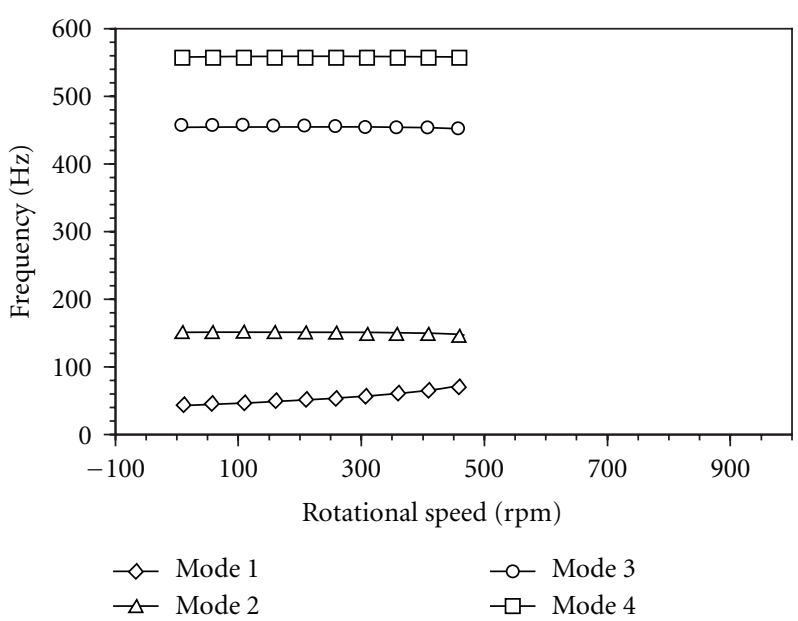

Figure 1: The first four frequencies of a wind turbine blade at different rotational speeds.

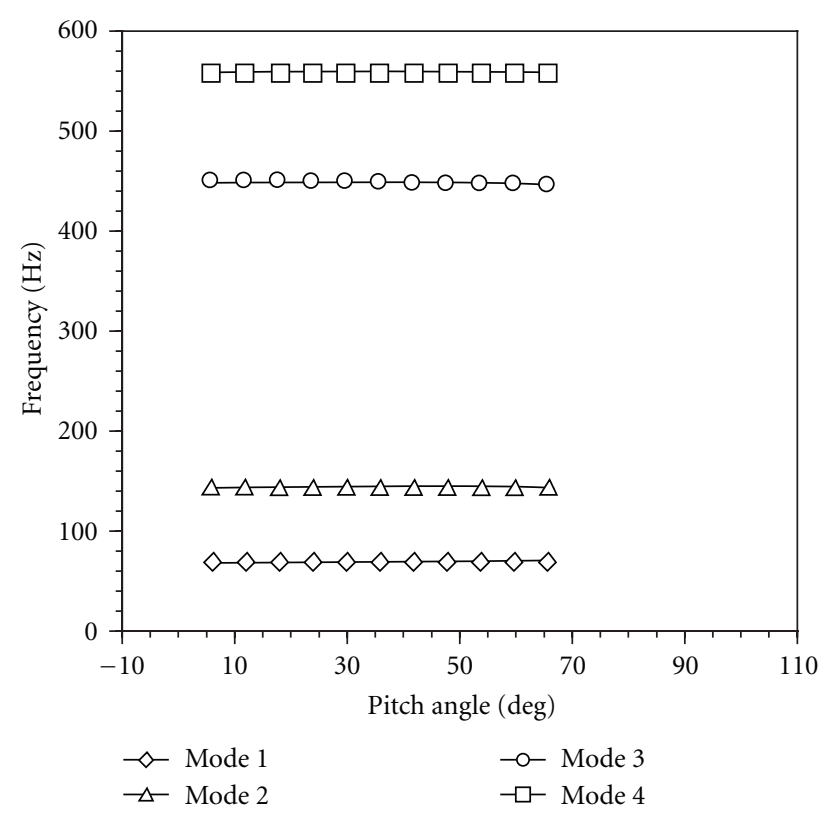

FIGURE 2: The first four frequencies of a wind turbine blade at different pitch angles.

\section{Dynamic Analysis of Wind Turbine Blades}

The kinetic energy of rotating wind turbine blades can be derived as

$$
\begin{gathered}
T^{e}=\sum_{k=1}^{3} \frac{1}{2} \int_{0}^{L} \rho A\left(\left(\frac{\partial u_{k}}{\partial t}\right)^{2}+\left(\frac{\partial v_{k}}{\partial t}\right)^{2}+\left(\Omega v_{k} \cos \theta\right)^{2}\right. \\
\left.+\left(\Omega u_{k} \sin \theta\right)^{2}\right) d r
\end{gathered}
$$


where $\Omega$ is hub rotational speed, $A$ is cross-sectional area, $\theta$ is pitch angle, $\rho$ is blade density, and $L$ is blade length. The corresponding strain energy of rotating blades is

$$
\begin{aligned}
& U^{e}=\sum_{k=1}^{3}( \frac{1}{2} \int_{0}^{L} E\left(I_{y y}\left(\frac{\partial u_{k}}{\partial r}\right)^{2}+2 I_{x y}\left(\frac{\partial u_{k}}{\partial r}\right)\left(\frac{\partial v_{k}}{\partial r}\right)\right. \\
&\left.+I_{x x}\left(\frac{\partial v_{k}}{\partial r}\right)^{2}\right) d r \\
&+\frac{1}{2} \int_{0}^{L} \int_{r}^{L} \rho A \Omega^{2}\left(r+\bar{r}_{0}\right) d r \\
&\left.\times\left(\left(\frac{\partial u_{k}}{\partial r}\right)^{2}+\left(\frac{\partial v_{k}}{\partial r}\right)^{2}\right) d r\right),
\end{aligned}
$$

where $\bar{r}_{0}$ is hub radius, $u_{1}(r, t)$ is the displacement of the first blade on the $x$-axis, $v_{1}(r, t)$ is the displacement of the first blade on the $y$-axis, $u_{2}(r, t)$ is the displacement of the second blade on the $x$-axis, $v_{2}(r, t)$ is the displacement of the second blade on the $y$-axis, $u_{3}(r, t)$ is the displacement of the third blade in the $x$-axis, $v_{3}(r, t)$ is the displacement of the third blade on the $y$-axis, and $E$ is Young's modulus. By examining the internal and external damping effects of blades, virtual work $\delta W^{e}$ in wind turbine blades can be derived as

$\delta W^{e}$

$$
\begin{aligned}
=\sum_{k=1}^{3}( & -\int_{0}^{L} C_{0} \frac{\partial u_{k}}{\partial t} \delta u_{k} d r-\int_{0}^{L} C_{1} \frac{\partial^{2} E I_{y y}}{\partial r^{2}} \frac{\partial^{3} u_{k}}{\partial t \partial r^{2}} \delta u_{k} d r \\
& -2 \int_{0}^{L} C_{1} \frac{\partial E I_{y y}}{\partial r} \frac{\partial^{4} u_{k}}{\partial t \partial r^{3}} \delta u_{k} d r \\
& -\int_{0}^{L} C_{1} E I_{y y} \frac{\partial^{5} u_{k}}{\partial t \partial r^{4}} \delta u_{k} d r \\
& -\int_{0}^{L} C_{0} \frac{\partial v_{k}}{\partial t} \delta v_{k} d r-\int_{0}^{L} C_{1} \frac{\partial^{2} E I_{x x}}{\partial r^{2}} \frac{\partial^{3} v_{k}}{\partial t \partial r^{2}} \delta v_{k} d r \\
& -2 \int_{0}^{L} C_{1} \frac{\partial E I_{x x}}{\partial r} \frac{\partial^{4} v_{k}}{\partial t \partial r^{3}} \delta v_{k} d r \\
& \left.-\int_{0}^{L} C_{1} E I_{x x} \frac{\partial^{5} v_{k}}{\partial t \partial r^{4}} \delta v_{k} d r\right),
\end{aligned}
$$

where $C_{0}$ and $C_{1}$ are external and internal damping coefficients of blades, respectively. Equations (6)-(8) are integrated into the Hamilton equation as follows:

$$
\int_{t_{1}}^{t_{2}}\left(\delta T^{e}-\delta U^{e}+\delta W^{e}\right) d t=0 .
$$

This leads to the following equations for the motion of the blades:

$$
\begin{aligned}
& \frac{\partial^{2} E I_{y y}}{\partial r^{2}} \frac{\partial^{2} u_{k}}{\partial r^{2}}+2 \frac{\partial E I_{y y}}{\partial r} \frac{\partial^{3} u_{k}}{\partial r^{3}}+E I_{y y} \frac{\partial^{4} u_{k}}{\partial r^{4}}+\frac{\partial^{2} E I_{x y}}{\partial r^{2}} \frac{\partial^{2} v_{k}}{\partial r^{2}} \\
& +2 \frac{\partial E I_{x y}}{\partial r} \frac{\partial^{3} v_{k}}{\partial r^{3}}+E I_{x y} \frac{\partial^{4} v_{k}}{\partial r^{4}}-\rho A \Omega^{2} u_{k} \sin ^{2} \theta \\
& -\rho \Omega^{2} \frac{\partial}{\partial r}\left(\int_{r}^{L} A\left(r+\bar{r}_{0}\right) d r\right) \frac{\partial u_{k}}{\partial r} \\
& -\rho \Omega^{2}\left(\int_{r}^{L} A\left(r+\bar{r}_{0}\right) d r\right) \frac{\partial^{2} u_{k}}{\partial r^{2}} \\
& +C_{0} \frac{\partial u_{k}}{\partial t}+C_{1} \frac{\partial^{2} E I_{y y}}{\partial r^{2}} \frac{\partial^{3} u_{k}}{\partial r^{2} \partial t}+2 C_{1} \frac{\partial E I_{y y}}{\partial r} \frac{\partial^{4} u_{k}}{\partial r^{3} \partial t} \\
& +C_{1} E I_{y y} \frac{\partial^{5} u_{k}}{\partial r^{4} \partial t}+C_{1} \frac{\partial^{2} E I_{x y}}{\partial r^{2}} \frac{\partial^{3} v_{k}}{\partial r^{2} \partial t}+2 C_{1} \frac{\partial E I_{x y}}{\partial r} \frac{\partial^{4} v_{k}}{\partial r^{3} \partial t} \\
& +C_{1} E I_{x y} \frac{\partial^{5} v_{k}}{\partial r^{4} \partial t}+\rho A \frac{\partial^{2} u_{k}}{\partial t^{2}}=0 \quad \text { for } k=1,2,3, \\
& \frac{\partial^{2} E I_{x x}}{\partial r^{2}} \frac{\partial^{2} v_{k}}{\partial r^{2}}+2 \frac{\partial E I_{x x}}{\partial r} \frac{\partial^{3} v_{k}}{\partial r^{3}}+E I_{x x} \frac{\partial^{4} v_{k}}{\partial r^{4}}+\frac{\partial^{2} E I_{x y}}{\partial r^{2}} \frac{\partial^{2} u_{k}}{\partial r^{2}} \\
& +2 \frac{\partial E I_{x y}}{\partial r} \frac{\partial^{3} u_{k}}{\partial r^{3}}+E I_{x y} \frac{\partial^{4} u_{k}}{\partial r^{4}}-\rho A \Omega^{2} v_{k} \cos ^{2} \theta \\
& -\rho \Omega^{2} \frac{\partial}{\partial r}\left(\int_{r}^{L} A\left(r+\bar{r}_{0}\right) d r\right) \frac{\partial v_{k}}{\partial r} \\
& -\rho \Omega^{2}\left(\int_{r}^{L} A\left(r+\bar{r}_{0}\right) d r\right) \frac{\partial^{2} v_{k}}{\partial r^{2}} \\
& +C_{0} \frac{\partial v_{k}}{\partial t}+C_{1} \frac{\partial^{2} E I_{x x}}{\partial r^{2}} \frac{\partial^{3} v_{k}}{\partial r^{2} \partial t}+2 C_{1} \frac{\partial E I_{x x}}{\partial r} \frac{\partial^{4} v_{k}}{\partial r^{3} \partial t} \\
& +C_{1} E I_{x x} \frac{\partial^{5} v_{k}}{\partial r^{4} \partial t}+C_{1} \frac{\partial^{2} E I_{x y}}{\partial r^{2}} \frac{\partial^{3} u_{k}}{\partial r^{2} \partial t}+2 C_{1} \frac{\partial E I_{x y}}{\partial r} \frac{\partial^{4} u_{k}}{\partial r^{3} \partial t} \\
& +C_{1} E I_{x y} \frac{\partial^{5} u_{k}}{\partial r^{4} \partial t}+\rho A \frac{\partial^{2} v_{k}}{\partial t^{2}}=0 \quad \text { for } k=1,2,3 \text {. }
\end{aligned}
$$

The following equations are the corresponding boundary conditions:

$$
\begin{gathered}
u_{k}(0, t)=0 \quad \text { for } k=1,2,3, \\
\frac{\partial u_{k}(0, t)}{\partial r}=0 \quad \text { for } k=1,2,3, \\
E I_{y y} \frac{\partial^{2} u_{k}(L, t)}{\partial r^{2}}=0 \quad \text { for } k=1,2,3, \\
\frac{\partial}{\partial r}\left(E I_{y y} \frac{\partial^{2} u_{k}(L, t)}{\partial r^{2}}\right)=0 \quad \text { for } k=1,2,3, \\
v_{k}(0, t)=0 \quad \text { for } k=1,2,3,
\end{gathered}
$$




$$
\begin{gathered}
\frac{\partial v_{k}(0, t)}{\partial r}=0 \quad \text { for } k=1,2,3 \\
E I_{x x} \frac{\partial^{2} v_{k}(L, t)}{\partial r^{2}}=0 \quad \text { for } k=1,2,3, \\
\frac{\partial}{\partial r}\left(E I_{x x} \frac{\partial^{2} v_{k}(L, t)}{\partial r^{2}}\right)=0 \quad \text { for } k=1,2,3 .
\end{gathered}
$$

A solution is thus assumed as

$$
\begin{array}{ll}
u_{k}=U_{k}(r) e^{\lambda t} & \text { for } k=1,2,3, \\
v_{k}=V_{k}(r) e^{\lambda t} & \text { for } k=1,2,3 .
\end{array}
$$

This yields the following equations for the motion of blades:

$$
\begin{aligned}
\frac{d^{2} E I_{y y}}{d r^{2}} \frac{d^{2} U_{k}}{d r^{2}}+2 \frac{d E I_{y y}}{d r} \frac{d^{3} U_{k}}{d r^{3}}+E I_{y y} \frac{d^{4} U_{k}}{d r^{4}}+\frac{d^{2} E I_{x y}}{d r^{2}} \frac{d^{2} V_{k}}{d r^{2}} \\
+2 \frac{d E I_{x y}}{d r} \frac{d^{3} V_{k}}{d r^{3}}+E I_{x y} \frac{d^{4} V_{k}}{d r^{4}}-\rho A \Omega^{2} U_{k} \sin ^{2} \theta \\
-\rho \Omega^{2} \frac{d}{d r}\left(\int_{r}^{L} A\left(r+\bar{r}_{0}\right) d r\right) \frac{d U_{k}}{d r} \\
-\rho \Omega^{2}\left(\int_{r}^{L} A\left(r+\bar{r}_{0}\right) d r\right) \frac{d^{2} U_{k}}{d r^{2}} \\
+\lambda C_{0} U_{k}+\lambda C_{1} \frac{d^{2} E I_{y y}}{d r^{2}} \frac{d^{2} U_{k}}{d r^{2}}+2 \lambda C_{1} \frac{d E I_{y y}}{d r} \frac{d^{3} U_{k}}{d r^{3}} \\
+\lambda C_{1} E I_{y y} \frac{d^{4} U_{k}}{d r^{4}}+\lambda C_{1} \frac{d^{2} E I_{x y}}{d r^{2}} \frac{d^{2} V_{k}}{d r^{2}} \\
+2 \lambda C_{1} \frac{d E I_{x y}}{d r} \frac{d^{3} V_{k}}{d r^{3}}+\lambda C_{1} E I_{x y} \frac{d^{4} V_{k}}{d r^{4}} \\
+\lambda^{2} \rho A U_{k}=0 \\
+2 \frac{d E I_{x y}}{d r} \frac{d^{3} U_{k}}{d r^{3}}+E I_{x y} \frac{d^{4} U_{k}}{d r^{4}}-\rho A \Omega^{2} V_{k} \cos ^{2} \theta \\
\frac{d^{2} E I_{x x}}{d r^{2}} \frac{d^{2} V_{k}}{d r^{2}}+2 \frac{d E I_{x x}}{d r} \frac{d^{3} V_{k}}{d r^{3}}+E I_{x x} \frac{d^{4} V_{k}}{d r^{4}}+\frac{d^{2} E I_{x y}}{d r^{2}} \frac{d^{2} U_{k}}{d r^{2}} \\
+3,
\end{aligned}
$$

$$
\begin{aligned}
& -\rho \Omega^{2} \frac{d}{d r}\left(\int_{r}^{L} A\left(r+\bar{r}_{0}\right) d r\right) \frac{d V_{k}}{d r} \\
& -\rho \Omega^{2}\left(\int_{r}^{L} A\left(r+\bar{r}_{0}\right) d r\right) \frac{d^{2} V_{k}}{d r^{2}} \\
& +\lambda C_{0} \frac{d V_{k}}{d t}+\lambda C_{1} \frac{d^{2} E I_{x x}}{d r^{2}} \frac{d^{2} V_{k}}{d r^{2}}+2 \lambda C_{1} \frac{d E I_{x x}}{d r} \frac{d^{3} V_{k}}{d r^{3}} \\
& +\lambda C_{1} E I_{x x} \frac{d^{4} V_{k}}{d r^{4}}+\lambda C_{1} \frac{d^{2} E I_{x y}}{d r^{2}} \frac{d^{2} U_{k}}{d r^{2}}+2 \lambda C_{1} \frac{d E I_{x y}}{d r} \frac{d^{3} U_{k}}{d r^{3}} \\
& +\lambda C_{1} E I_{x y} \frac{d^{4} U_{k}}{d r^{4}}+\lambda^{2} \rho A V_{k}=0 \quad \text { for } k=1,2,3 .
\end{aligned}
$$

The corresponding boundary conditions are as follows:

$$
\begin{gathered}
U_{k}(0)=0 \quad \text { for } k=1,2,3, \\
\frac{d U_{k}(0)}{d r}=0 \quad \text { for } k=1,2,3, \\
E I_{y y} \frac{d^{2} U_{k}(L)}{d r^{2}}=0 \quad \text { for } k=1,2,3, \\
\frac{d}{d r}\left(E I_{y y} \frac{d^{2} U_{k}(L)}{d r^{2}}\right)=0 \quad \text { for } k=1,2,3, \\
\frac{V_{k}(0)=0}{d V_{k}(0)} d=0 \quad \text { for } k=1,2,3, \\
E I_{x x} \frac{d^{2} V_{k}(L)}{d r^{2}}=0 \quad \text { for } k=1,2,3, \\
\frac{d}{d r}\left(E I_{x x} \frac{d^{2} V_{k}(L)}{d r^{2}}\right)=0 \quad \text { for } k=1,2,3 .
\end{gathered}
$$

By employing the radial basis function approach, (2) and (3) can be substituted into (10). The equations of motion of wind turbine blades can be rearranged in the following matrix form:

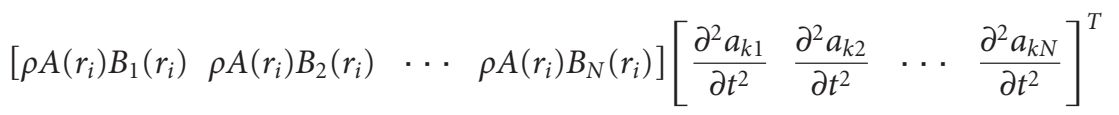

$$
\begin{aligned}
& +\left[\begin{array}{lllll}
C_{0} B_{1}\left(r_{i}\right) & C_{0} B_{2}\left(r_{i}\right) & \cdots & C_{0} B_{N}\left(r_{i}\right)
\end{array}\right]\left[\begin{array}{llll}
\frac{\partial a_{k 1}}{\partial t} & \frac{\partial a_{k 2}}{\partial t} & \cdots & \frac{\partial a_{k N}}{\partial t}
\end{array}\right]^{T}
\end{aligned}
$$

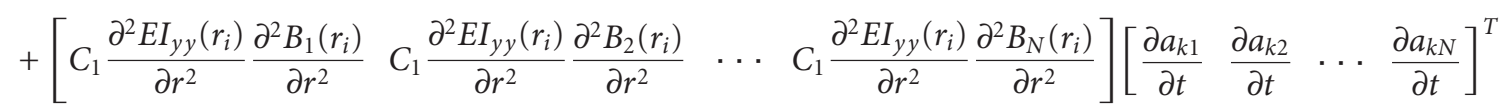

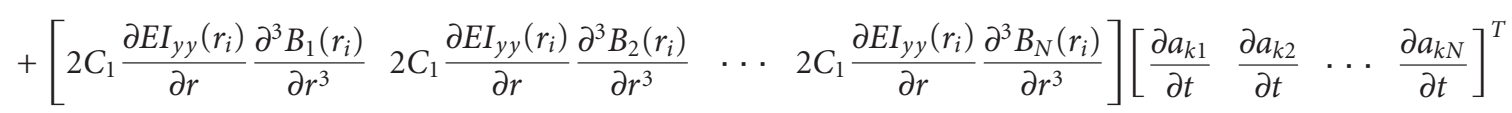

$$
\begin{aligned}
& +\left[\begin{array}{lllll}
C_{1} E I_{y y}\left(r_{i}\right) \frac{\partial^{4} B_{1}\left(r_{i}\right)}{\partial r^{4}} & C_{1} E I_{y y}\left(r_{i}\right) \frac{\partial^{4} B_{2}\left(r_{i}\right)}{\partial r^{4}} & \cdots & C_{1} E I_{y y}\left(r_{i}\right) \frac{\partial^{4} B_{N}\left(r_{i}\right)}{\partial r^{4}}
\end{array}\right]\left[\begin{array}{llll}
\frac{\partial a_{k 1}}{\partial t} & \frac{\partial a_{k 2}}{\partial t} & \cdots & \frac{\partial a_{k N}}{\partial t}
\end{array}\right]^{T}
\end{aligned}
$$


Advances in Acoustics and Vibration

5

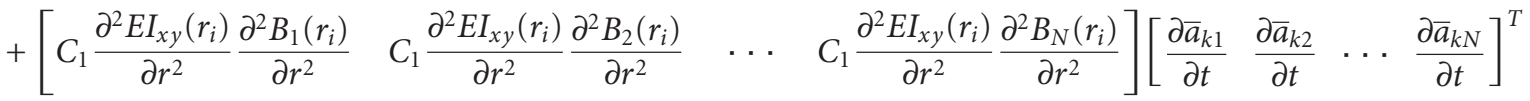

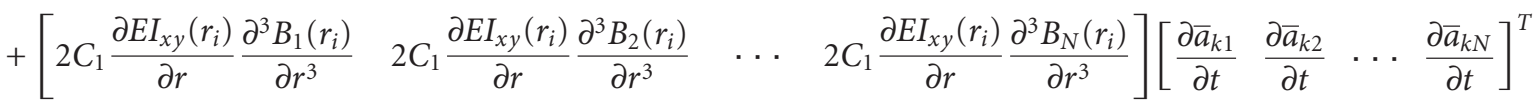

$$
\begin{aligned}
& +\left[\begin{array}{llll}
C_{1} E I_{x y}\left(r_{i}\right) \frac{\partial^{4} B_{1}\left(r_{i}\right)}{\partial r^{4}} & C_{1} E I_{x y}\left(r_{i}\right) \frac{\partial^{4} B_{2}\left(r_{i}\right)}{\partial r^{4}} & \cdots & C_{1} E I_{x y}\left(r_{i}\right) \frac{\partial^{4} B_{N}\left(r_{i}\right)}{\partial r^{4}}
\end{array}\right]\left[\begin{array}{llll}
\frac{\partial \bar{a}_{k 1}}{\partial t} & \frac{\partial \bar{a}_{k 2}}{\partial t} & \cdots & \frac{\partial \bar{a}_{k N}}{\partial t}
\end{array}\right]^{T} \\
& +\left[\begin{array}{llll}
\frac{\partial^{2} E I_{y y}\left(r_{i}\right)}{\partial r^{2}} \frac{\partial^{2} B_{1}\left(r_{i}\right)}{\partial r^{2}} & \frac{\partial^{2} E I_{y y}\left(r_{i}\right)}{\partial r^{2}} \frac{\partial^{2} B_{2}\left(r_{i}\right)}{\partial r^{2}} & \cdots & \frac{\partial^{2} E I_{y y}\left(r_{i}\right)}{\partial r^{2}} \frac{\partial^{2} B_{N}\left(r_{i}\right)}{\partial r^{2}}
\end{array}\right]\left[\begin{array}{llll}
a_{k 1} & a_{k 2} & \cdots & a_{k N}
\end{array}\right]^{T} \\
& +\left[\begin{array}{llll}
2 \frac{\partial E I_{y y}\left(r_{i}\right)}{\partial r} \frac{\partial^{3} B_{1}\left(r_{i}\right)}{\partial r^{3}} & 2 \frac{\partial E I_{y y}\left(r_{i}\right)}{\partial r} \frac{\partial^{3} B_{2}\left(r_{i}\right)}{\partial r^{3}} & \cdots & 2 \frac{\partial E I_{y y}\left(r_{i}\right)}{\partial r} \frac{\partial^{3} B_{N}\left(r_{i}\right)}{\partial r^{3}}
\end{array}\right]\left[\begin{array}{llll}
a_{k 1} & a_{k 2} & \cdots & a_{k N}
\end{array}\right]^{T} \\
& +\left[\begin{array}{llll}
E I_{y y}\left(r_{i}\right) \frac{\partial^{4} B_{1}\left(r_{i}\right)}{\partial r^{4}} & E I_{y y}\left(r_{i}\right) \frac{\partial^{4} B_{2}\left(r_{i}\right)}{\partial r^{4}} & \cdots & E I_{y y}\left(r_{i}\right) \frac{\partial^{4} B_{N}\left(r_{i}\right)}{\partial r^{4}}
\end{array}\right]\left[\begin{array}{llll}
a_{k 1} & a_{k 2} & \cdots & a_{k N}
\end{array}\right]^{T} \\
& +\left[\frac{\partial^{2} E I_{x y}\left(r_{i}\right)}{\partial r^{2}} \frac{\partial^{2} B_{1}\left(r_{i}\right)}{\partial r^{2}} \quad \frac{\partial^{2} E I_{x y}\left(r_{i}\right)}{\partial r^{2}} \frac{\partial^{2} B_{2}\left(r_{i}\right)}{\partial r^{2}} \cdots \frac{\partial^{2} E I_{x y}\left(r_{i}\right)}{\partial r^{2}} \frac{\partial^{2} B_{N}\left(r_{i}\right)}{\partial r^{2}}\right]\left[\begin{array}{llll}
\bar{a}_{k 1} & \bar{a}_{k 2} & \cdots & \bar{a}_{k N}
\end{array}\right]^{T} \\
& +\left[\begin{array}{llll}
2 \frac{\partial E I_{x y}\left(r_{i}\right)}{\partial r} \frac{\partial^{3} B_{1}\left(r_{i}\right)}{\partial r^{3}} & 2 \frac{\partial E I_{x y}\left(r_{i}\right)}{\partial r} \frac{\partial^{3} B_{2}\left(r_{i}\right)}{\partial r^{3}} & \cdots & 2 \frac{\partial E I_{x y}\left(r_{i}\right)}{\partial r} \frac{\partial^{3} B_{N}\left(r_{i}\right)}{\partial r^{3}}
\end{array}\right]\left[\begin{array}{llll}
\bar{a}_{k 1} & \bar{a}_{k 2} & \cdots & \bar{a}_{k N}
\end{array}\right]^{T} \\
& +\left[\begin{array}{llll}
E I_{x y}\left(r_{i}\right) \frac{\partial^{4} B_{1}\left(r_{i}\right)}{\partial r^{4}} & E I_{x y}\left(r_{i}\right) \frac{\partial^{4} B_{2}\left(r_{i}\right)}{\partial r^{4}} & \cdots & E I_{x y}\left(r_{i}\right) \frac{\partial^{4} B_{N}\left(r_{i}\right)}{\partial r^{4}}
\end{array}\right]\left[\begin{array}{llll}
\bar{a}_{k 1} & \bar{a}_{k 2} & \cdots & \bar{a}_{k N}
\end{array}\right]^{T} \\
& -\left[\begin{array}{llll}
\rho A(r) \Omega^{2}(\sin \theta)^{2} B_{1}\left(r_{i}\right) & \rho A(r) \Omega^{2}(\sin \theta)^{2} B_{2}\left(r_{i}\right) & \cdots & \rho A(r) \Omega^{2}(\sin \theta)^{2} B_{N}\left(r_{i}\right)
\end{array}\right]\left[\begin{array}{llll}
a_{k 1} & a_{k 2} & \cdots & a_{k N}
\end{array}\right]^{T}
\end{aligned}
$$

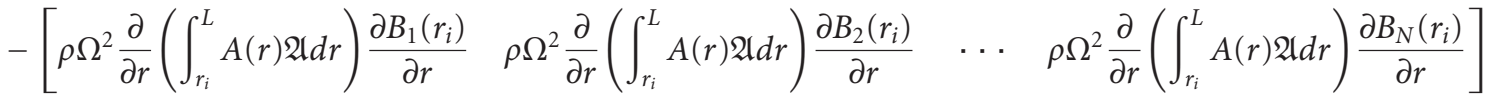

$$
\begin{aligned}
& \times\left[\begin{array}{llll}
a_{k 1} & a_{k 2} & \cdots & a_{k N}
\end{array}\right]^{T}
\end{aligned}
$$

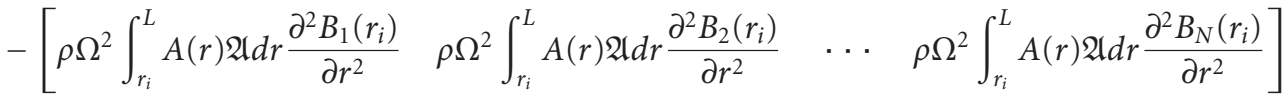

$$
\begin{aligned}
& \times\left[\begin{array}{llll}
a_{k 1} & a_{k 2} & \cdots & a_{k N}
\end{array}\right]^{T}=[0], \quad \text { for } i=1,2, \ldots, N, k=1,2,3, \\
& {\left[\begin{array}{llll}
\rho A\left(r_{i}\right) B_{1}\left(r_{i}\right) & \rho A\left(r_{i}\right) B_{2}\left(r_{i}\right) & \cdots & \rho A\left(r_{i}\right) B_{N}\left(r_{i}\right)
\end{array}\right]\left[\begin{array}{llll}
\frac{\partial^{2} \bar{a}_{k 1}}{\partial t^{2}} & \frac{\partial^{2} \bar{a}_{k 2}}{\partial t^{2}} & \cdots & \frac{\partial^{2} \bar{a}_{k N}}{\partial t^{2}}
\end{array}\right]^{T}} \\
& +\left[\begin{array}{llll}
C_{0} B_{1}\left(r_{i}\right) & C_{0} B_{2}\left(r_{i}\right) & \cdots & C_{0} B_{N}\left(r_{i}\right)
\end{array}\right]\left[\begin{array}{llll}
\frac{\partial \bar{a}_{k 1}}{\partial t} & \frac{\partial \bar{a}_{k 2}}{\partial t} & \cdots & \frac{\partial \bar{a}_{k N}}{\partial t}
\end{array}\right]^{T}
\end{aligned}
$$

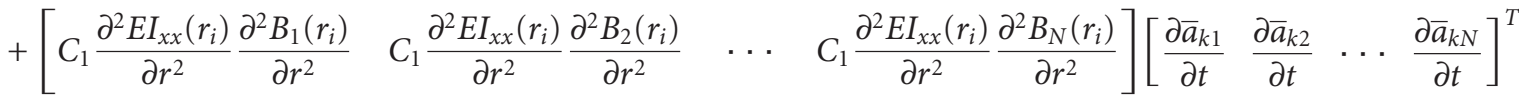

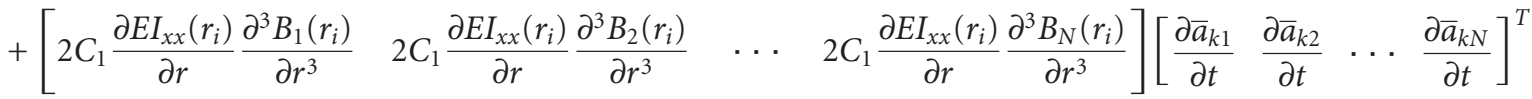

$$
\begin{aligned}
& +\left[\begin{array}{llll}
C_{1} E I_{x x}\left(r_{i}\right) \frac{\partial^{4} B_{1}\left(r_{i}\right)}{\partial r^{4}} & C_{1} E I_{x x}\left(r_{i}\right) \frac{\partial^{4} B_{2}\left(r_{i}\right)}{\partial r^{4}} & \cdots & C_{1} E I_{x x}\left(r_{i}\right) \frac{\partial^{4} B_{N}\left(r_{i}\right)}{\partial r^{4}}
\end{array}\right]\left[\begin{array}{llll}
\frac{\partial \bar{a}_{k 1}}{\partial t} & \frac{\partial \bar{a}_{k 2}}{\partial t} & \cdots & \frac{\partial \bar{a}_{k N}}{\partial t}
\end{array}\right]^{T}
\end{aligned}
$$

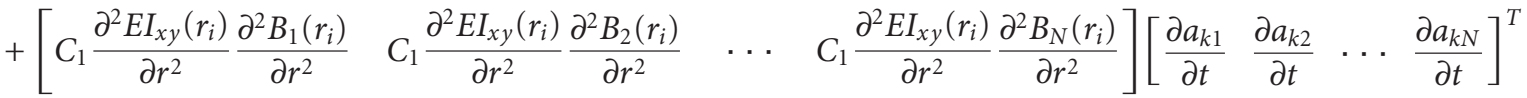




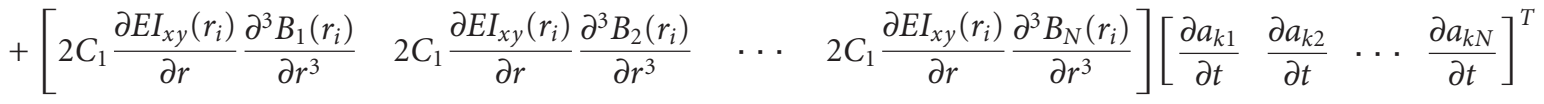

$$
\begin{aligned}
& +\left[\begin{array}{llll}
C_{1} E I_{x y}\left(r_{i}\right) \frac{\partial^{4} B_{1}\left(r_{i}\right)}{\partial r^{4}} & C_{1} E I_{x y}\left(r_{i}\right) \frac{\partial^{4} B_{2}\left(r_{i}\right)}{\partial r^{4}} & \cdots & C_{1} E I_{x y}\left(r_{i}\right) \frac{\partial^{4} B_{N}\left(r_{i}\right)}{\partial r^{4}}
\end{array}\right]\left[\begin{array}{llll}
\frac{\partial a_{k 1}}{\partial t} & \frac{\partial a_{k 2}}{\partial t} & \cdots & \frac{\partial a_{k N}}{\partial t}
\end{array}\right]^{T} \\
& +\left[\frac{\partial^{2} E I_{x x}\left(r_{i}\right)}{\partial r^{2}} \frac{\partial^{2} B_{1}\left(r_{i}\right)}{\partial r^{2}} \frac{\partial^{2} E I_{x x}\left(r_{i}\right)}{\partial r^{2}} \frac{\partial^{2} B_{2}\left(r_{i}\right)}{\partial r^{2}} \quad \ldots \quad \frac{\partial^{2} E I_{x x}\left(r_{i}\right)}{\partial r^{2}} \frac{\partial^{2} B_{N}\left(r_{i}\right)}{\partial r^{2}}\right]\left[\begin{array}{llll}
\bar{a}_{k 1} & \bar{a}_{k 2} & \cdots & \bar{a}_{k N}
\end{array}\right]^{T} \\
& +\left[\begin{array}{llll}
2 \frac{\partial E I_{x x}\left(r_{i}\right)}{\partial r} \frac{\partial^{3} B_{1}\left(r_{i}\right)}{\partial r^{3}} & 2 \frac{\partial E I_{x x}\left(r_{i}\right)}{\partial r} \frac{\partial^{3} B_{2}\left(r_{i}\right)}{\partial r^{3}} & \cdots & 2 \frac{\partial E I_{x x}\left(r_{i}\right)}{\partial r} \frac{\partial^{3} B_{N}\left(r_{i}\right)}{\partial r^{3}}
\end{array}\right]\left[\begin{array}{llll}
\bar{a}_{k 1} & \bar{a}_{k 2} & \cdots & \bar{a}_{k N}
\end{array}\right]^{T} \\
& +\left[\begin{array}{llll}
E I_{x x}\left(r_{i}\right) \frac{\partial^{4} B_{1}\left(r_{i}\right)}{\partial r^{4}} & E I_{x x}\left(r_{i}\right) \frac{\partial^{4} B_{2}\left(r_{i}\right)}{\partial r^{4}} & \cdots & E I_{x x}\left(r_{i}\right) \frac{\partial^{4} B_{N}\left(r_{i}\right)}{\partial r^{4}}
\end{array}\right]\left[\begin{array}{llll}
\bar{a}_{k 1} & \bar{a}_{k 2} & \cdots & \bar{a}_{k N}
\end{array}\right]^{T} \\
& +\left[\begin{array}{llll}
\frac{\partial^{2} E I_{x y}\left(r_{i}\right)}{\partial r^{2}} \frac{\partial^{2} B_{1}\left(r_{i}\right)}{\partial r^{2}} & \frac{\partial^{2} E I_{x y}\left(r_{i}\right)}{\partial r^{2}} \frac{\partial^{2} B_{2}\left(r_{i}\right)}{\partial r^{2}} & \cdots & \frac{\partial^{2} E I_{x y}\left(r_{i}\right)}{\partial r^{2}} \frac{\partial^{2} B_{N}\left(r_{i}\right)}{\partial r^{2}}
\end{array}\right]\left[\begin{array}{llll}
a_{k 1} & a_{k 2} & \cdots & a_{k N}
\end{array}\right]^{T} \\
& +\left[\begin{array}{lllll}
2 \frac{\partial E I_{x y}\left(r_{i}\right)}{\partial r} \frac{\partial^{3} B_{1}\left(r_{i}\right)}{\partial r^{3}} & 2 \frac{\partial E I_{x y}\left(r_{i}\right)}{\partial r} \frac{\partial^{3} B_{2}\left(r_{i}\right)}{\partial r^{3}} & \cdots & 2 \frac{\partial E I_{x y}\left(r_{i}\right)}{\partial r} \frac{\partial^{3} B_{N}\left(r_{i}\right)}{\partial r^{3}}
\end{array}\right]\left[\begin{array}{llll}
a_{k 1} & a_{k 2} & \cdots & a_{k N}
\end{array}\right]^{T} \\
& +\left[\begin{array}{llll}
E I_{x y}\left(r_{i}\right) \frac{\partial^{4} B_{1}\left(r_{i}\right)}{\partial r^{4}} & E I_{x y}\left(r_{i}\right) \frac{\partial^{4} B_{2}\left(r_{i}\right)}{\partial r^{4}} & \cdots & E I_{x y}\left(r_{i}\right) \frac{\partial^{4} B_{N}\left(r_{i}\right)}{\partial r^{4}}
\end{array}\right]\left[\begin{array}{llll}
a_{k 1} & a_{k 2} & \cdots & a_{k N}
\end{array}\right]^{T} \\
& -\left[\begin{array}{lllll}
\rho A(r) \Omega^{2}(\cos \theta)^{2} B_{1}\left(r_{i}\right) & \rho A(r) \Omega^{2}(\cos \theta)^{2} B_{2}\left(r_{i}\right) & \cdots & \rho A(r) \Omega^{2}(\cos \theta)^{2} B_{N}\left(r_{i}\right)
\end{array}\right]\left[\begin{array}{llll}
\bar{a}_{k 1} & \bar{a}_{k 2} & \cdots & \bar{a}_{k N}
\end{array}\right]^{T} \\
& -\left[\rho \Omega^{2} \frac{\partial}{\partial r}\left(\int_{r_{i}}^{L} A(r) \mathfrak{A} d r\right) \frac{\partial B_{1}\left(r_{i}\right)}{\partial r} \quad \rho \Omega^{2} \frac{\partial}{\partial r}\left(\int_{r_{i}}^{L} A(r) \mathfrak{A} d r\right) \frac{\partial B_{2}\left(r_{i}\right)}{\partial r} \quad \cdots \quad \rho \Omega^{2} \frac{\partial}{\partial r}\left(\int_{r_{i}}^{L} A(r) \mathfrak{A} d r\right) \frac{\partial B_{N}\left(r_{i}\right)}{\partial r}\right] \\
& \times\left[\begin{array}{llll}
\bar{a}_{k 1} & \bar{a}_{k 2} & \cdots & \bar{a}_{k N}
\end{array}\right]^{T}
\end{aligned}
$$

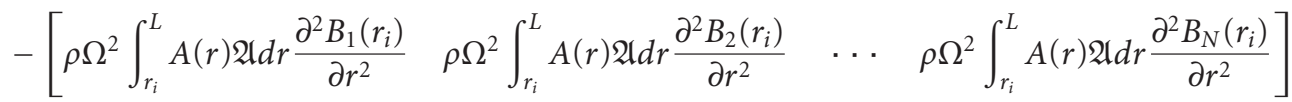

$$
\begin{aligned}
& \times\left[\begin{array}{llll}
\bar{a}_{k 1} & \bar{a}_{k 2} & \ldots & \bar{a}_{k N}
\end{array}\right]^{T}=[0] \text { for } i=1,2, \ldots, N, k=1,2,3 \text {, }
\end{aligned}
$$

where $\mathfrak{A}$ denotes $\left(r+\bar{r}_{0}\right)$.

Based on the radial basis function technique, (11) take the following discrete forms:

$$
\begin{aligned}
& {\left[\begin{array}{llll}
B_{1}\left(r_{1}\right) & B_{2}\left(r_{1}\right) & \cdots & B_{N}\left(r_{1}\right)
\end{array}\right]\left[\begin{array}{llll}
a_{k 1} & a_{k 2} & \cdots & a_{k N}
\end{array}\right]^{T}=[0] \text { for } k=1,2,3,} \\
& {\left[\begin{array}{llll}
\frac{\partial B_{1}\left(r_{1}\right)}{\partial r} & \frac{\partial B_{2}\left(r_{1}\right)}{\partial r} & \cdots & \frac{\partial B_{N}\left(r_{1}\right)}{\partial r}
\end{array}\right]\left[\begin{array}{llll}
a_{k 1} & a_{k 2} & \cdots & a_{k N}
\end{array}\right]^{T}=\left[\begin{array}{l}
0
\end{array}\right] \text { for } k=1,2,3,} \\
& {\left[\begin{array}{llll}
E I_{y y}\left(r_{N}\right) \frac{\partial^{2} B_{1}\left(r_{N}\right)}{\partial r^{2}} & E I_{y y}\left(r_{N}\right) \frac{\partial^{2} B_{2}\left(r_{N}\right)}{\partial r^{2}} & \cdots & E I_{y y}\left(r_{N}\right) \frac{\partial^{2} B_{N}\left(r_{N}\right)}{\partial r^{2}}
\end{array}\right]\left[\begin{array}{llll}
a_{k 1} & a_{k 2} & \cdots & a_{k N}
\end{array}\right]^{T}=[0] \quad \text { for } k=1,2,3 \text {, }} \\
& {\left[\begin{array}{llll}
\frac{\partial E I_{y y}\left(r_{N}\right)}{\partial r} \frac{\partial^{2} B_{1}\left(r_{N}\right)}{\partial r^{2}} & \frac{\partial E I_{y y}\left(r_{N}\right)}{\partial r} \frac{\partial^{2} B_{2}\left(r_{N}\right)}{\partial r^{2}} & \cdots & \frac{\partial E I_{y y}\left(r_{N}\right)}{\partial r} \frac{\partial^{2} B_{N}\left(r_{N}\right)}{\partial r^{2}}
\end{array}\right]\left[\begin{array}{lllll}
a_{k 1} & a_{k 2} & \cdots & a_{k N}
\end{array}\right]^{T}} \\
& +\left[\begin{array}{llll}
E I_{y y}\left(r_{N}\right) \frac{\partial^{3} B_{1}\left(r_{N}\right)}{\partial r^{3}} & E I_{y y}\left(r_{N}\right) \frac{\partial^{3} B_{2}\left(r_{N}\right)}{\partial r^{3}} & \cdots & E I_{y y}\left(r_{N}\right) \frac{\partial^{3} B_{N}\left(r_{N}\right)}{\partial r^{3}}
\end{array}\right] \\
& \times\left[\begin{array}{llll}
a_{k 1} & a_{k 2} & \cdots & a_{k N}
\end{array}\right]^{T}=[0] \text { for } k=1,2,3 \text {, }
\end{aligned}
$$




$$
\begin{aligned}
& {\left[\begin{array}{llllll}
B_{1}\left(r_{1}\right) & B_{2}\left(r_{1}\right) & \cdots & B_{N}\left(r_{1}\right)
\end{array}\right]\left[\begin{array}{llll}
\bar{a}_{k 1} & \bar{a}_{k 2} & \cdots & \bar{a}_{k N}
\end{array}\right]^{T}=[0] \quad \text { for } k=1,2,3,} \\
& {\left[\begin{array}{llll}
\frac{\partial B_{1}\left(r_{1}\right)}{\partial r} & \frac{\partial B_{2}\left(r_{1}\right)}{\partial r} & \cdots & \frac{\partial B_{N}\left(r_{1}\right)}{\partial r}
\end{array}\right]\left[\begin{array}{llll}
\bar{a}_{k 1} & \bar{a}_{k 2} & \cdots & \bar{a}_{k N}
\end{array}\right]^{T}=[0] \quad \text { for } k=1,2,3,} \\
& {\left[\begin{array}{llll}
E I_{x x}\left(r_{N}\right) \frac{\partial^{2} B_{1}\left(r_{N}\right)}{\partial r^{2}} & E I_{x x}\left(r_{N}\right) \frac{\partial^{2} B_{2}\left(r_{N}\right)}{\partial r^{2}} & \cdots & E I_{x x}\left(r_{N}\right) \frac{\partial^{2} B_{N}\left(r_{N}\right)}{\partial r^{2}}
\end{array}\right]\left[\begin{array}{llll}
\bar{a}_{k 1} & \bar{a}_{k 2} & \cdots & \bar{a}_{k N}
\end{array}\right]^{T}=[0], \quad \text { for } k=1,2,3,} \\
& {\left[\begin{array}{llll}
\frac{\partial E I_{x x}\left(r_{N}\right)}{\partial r} \frac{\partial^{2} B_{1}\left(r_{N}\right)}{\partial r^{2}} & \frac{\partial E I_{x x}\left(r_{N}\right)}{\partial r} \frac{\partial^{2} B_{2}\left(r_{N}\right)}{\partial r^{2}} & \cdots & \frac{\partial E I_{x x}\left(r_{N}\right)}{\partial r} \frac{\partial^{2} B_{N}\left(r_{N}\right)}{\partial r^{2}}
\end{array}\right]\left[\begin{array}{llll}
\bar{a}_{k 1} & \bar{a}_{k 2} & \cdots & \bar{a}_{k N}
\end{array}\right]^{T}} \\
& +\left[\begin{array}{llll}
E I_{x x}\left(r_{N}\right) \frac{\partial^{3} B_{N}\left(r_{N}\right)}{\partial r^{3}} & E I_{x x}\left(r_{N}\right) \frac{\partial^{3} B_{2}\left(r_{N}\right)}{\partial r^{3}} & \cdots & E I_{x x}\left(r_{N}\right) \frac{\partial^{3} B_{N}\left(r_{N}\right)}{\partial r^{3}}
\end{array}\right] \\
& \times\left[\begin{array}{llll}
\bar{a}_{k 1} & \bar{a}_{k 2} & \cdots & \bar{a}_{k N}
\end{array}\right]^{T}=[0] \quad \text { for } k=1,2,3 .
\end{aligned}
$$

By applying the radial basis function approach, (4) and (5) are substituted into (13). The following equations are then yielded

$$
\begin{aligned}
& {\left[\begin{array}{lllll}
\lambda^{2} \rho A\left(r_{i}\right) B_{1}\left(r_{i}\right) & \lambda^{2} \rho A\left(r_{i}\right) B_{2}\left(r_{i}\right) & \cdots & \lambda^{2} \rho A\left(r_{i}\right) B_{N}\left(r_{i}\right)
\end{array}\right]\left[\begin{array}{llll}
b_{k 1} & b_{k 2} & \cdots & b_{k N}
\end{array}\right]^{T}} \\
& +\left[\begin{array}{lllll}
\lambda C_{0} B_{1}\left(r_{i}\right) & \lambda C_{0} B_{2}\left(r_{i}\right) & \cdots & \lambda C_{0} B_{N}\left(r_{i}\right)
\end{array}\right]\left[\begin{array}{llll}
b_{k 1} & b_{k 2} & \cdots & b_{k N}
\end{array}\right]^{T} \\
& +\left[\begin{array}{llllll}
\lambda C_{1} \frac{d^{2} E I_{y y}\left(r_{i}\right)}{d r^{2}} \frac{d^{2} B_{1}\left(r_{i}\right)}{d r^{2}} & \lambda C_{1} \frac{d^{2} E I_{y y}\left(r_{i}\right)}{d r^{2}} \frac{d^{2} B_{2}\left(r_{i}\right)}{d r^{2}} & \cdots & \lambda C_{1} \frac{d^{2} E I_{y y}\left(r_{i}\right)}{d r^{2}} \frac{d^{2} B_{N}\left(r_{i}\right)}{d r^{2}}
\end{array}\right]\left[\begin{array}{llll}
b_{k 1} & b_{k 2} & \cdots & b_{k N}
\end{array}\right]^{T} \\
& +\left[2 \lambda C_{1} \frac{d E I_{y y}\left(r_{i}\right)}{d r} \frac{d^{3} B_{1}\left(r_{i}\right)}{d r^{3}} \quad 2 \lambda C_{1} \frac{d E I_{y y}\left(r_{i}\right)}{d r} \frac{d^{3} B_{2}\left(r_{i}\right)}{d r^{3}} \quad \cdots \quad 2 \lambda C_{1} \frac{d E I_{y y}\left(r_{i}\right)}{d r} \frac{d^{3} B_{N}\left(r_{i}\right)}{d r^{3}}\right]\left[\begin{array}{llll}
b_{k 1} & b_{k 2} & \cdots & b_{k N}
\end{array}\right]^{T} \\
& +\left[\begin{array}{lllll}
\lambda C_{1} E I_{y y}\left(r_{i}\right) \frac{d^{4} B_{1}\left(r_{i}\right)}{d r^{4}} & \lambda C_{1} E I_{y y}\left(r_{i}\right) \frac{d^{4} B_{2}\left(r_{i}\right)}{d r^{4}} & \cdots & \lambda C_{1} E I_{y y}\left(r_{i}\right) \frac{d^{4} B_{N}\left(r_{i}\right)}{d r^{4}}
\end{array}\right]\left[\begin{array}{llll}
b_{k 1} & b_{k 2} & \cdots & b_{k N}
\end{array}\right]^{T} \\
& +\left[\begin{array}{lllll}
\lambda C_{1} \frac{d^{2} E I_{x y}\left(r_{i}\right)}{d r^{2}} \frac{d^{2} B_{1}\left(r_{i}\right)}{d r^{2}} & \lambda C_{1} \frac{d^{2} E I_{x y}\left(r_{i}\right)}{d r^{2}} \frac{d^{2} B_{2}\left(r_{i}\right)}{d r^{2}} & \cdots & \lambda C_{1} \frac{d^{2} E I_{x y}\left(r_{i}\right)}{d r^{2}} \frac{d^{2} B_{N}\left(r_{i}\right)}{d r^{2}}
\end{array}\right]\left[\begin{array}{llll}
\bar{b}_{k 1} & \bar{b}_{k 2} & \cdots & \bar{b}_{k N}
\end{array}\right]^{T} \\
& +\left[\begin{array}{lllll}
2 \lambda C_{1} \frac{d E I_{x y}\left(r_{i}\right)}{d r} \frac{d^{3} B_{1}\left(r_{i}\right)}{d r^{3}} & 2 \lambda C_{1} \frac{d E I_{x y}\left(r_{i}\right)}{d r} \frac{d^{3} B_{2}\left(r_{i}\right)}{d r^{3}} & \cdots & 2 \lambda C_{1} \frac{d E I_{x y}\left(r_{i}\right)}{d r} \frac{d^{3} B_{N}\left(r_{i}\right)}{d r^{3}}
\end{array}\right]\left[\begin{array}{llll}
\bar{b}_{k 1} & \bar{b}_{k 2} & \cdots & \bar{b}_{k N}
\end{array}\right]^{T} \\
& +\left[\begin{array}{lllll}
\lambda C_{1} E I_{x y}\left(r_{i}\right) \frac{d^{4} B_{1}\left(r_{i}\right)}{d r^{4}} & \lambda C_{1} E I_{x y}\left(r_{i}\right) \frac{d^{4} B_{2}\left(r_{i}\right)}{d r^{4}} & \cdots & \lambda C_{1} E I_{x y}\left(r_{i}\right) \frac{d^{4} B_{N}\left(r_{i}\right)}{d r^{4}}
\end{array}\right]\left[\begin{array}{llll}
\bar{b}_{k 1} & \bar{b}_{k 2} & \cdots & \bar{b}_{k N}
\end{array}\right]^{T} \\
& +\left[\begin{array}{lllll}
\frac{d^{2} E I_{y y}\left(r_{i}\right)}{d r^{2}} \frac{d^{2} B_{1}\left(r_{i}\right)}{d r^{2}} & \frac{d^{2} E I_{y y}\left(r_{i}\right)}{d r^{2}} \frac{d^{2} B_{2}\left(r_{i}\right)}{d r^{2}} & \cdots & \frac{d^{2} E I_{y y}\left(r_{i}\right)}{d r^{2}} \frac{d^{2} B_{N}\left(r_{i}\right)}{d r^{2}}
\end{array}\right]\left[\begin{array}{llll}
b_{k 1} & b_{k 2} & \cdots & b_{k N}
\end{array}\right]^{T} \\
& +\left[\begin{array}{lllll}
2 \frac{d E I_{y y}\left(r_{i}\right)}{d r} \frac{d^{3} B_{1}\left(r_{i}\right)}{d r^{3}} & 2 \frac{d E I_{y y}\left(r_{i}\right)}{d r} \frac{d^{3} B_{2}\left(r_{i}\right)}{d r^{3}} & \cdots & 2 \frac{d E I_{y y}\left(r_{i}\right)}{d r} \frac{d^{3} B_{N}\left(r_{i}\right)}{d r^{3}}
\end{array}\right]\left[\begin{array}{llll}
b_{k 1} & b_{k 2} & \cdots & b_{k N}
\end{array}\right]^{T} \\
& +\left[\begin{array}{llll}
E I_{y y}\left(r_{i}\right) \frac{d^{4} B_{1}\left(r_{i}\right)}{d r^{4}} & E I_{y y}\left(r_{i}\right) \frac{d^{4} B_{2}\left(r_{i}\right)}{d r^{4}} & \cdots & E I_{y y}\left(r_{i}\right) \frac{d^{4} B_{N}\left(r_{i}\right)}{d r^{4}}
\end{array}\right]\left[\begin{array}{llll}
b_{k 1} & b_{k 2} & \cdots & b_{k N}
\end{array}\right]^{T} \\
& +\left[\begin{array}{llll}
\frac{d^{2} E I_{x y}\left(r_{i}\right)}{d r^{2}} \frac{d^{2} B_{1}\left(r_{i}\right)}{d r^{2}} & \frac{d^{2} E I_{x y}\left(r_{i}\right)}{d r^{2}} \frac{d^{2} B_{2}\left(r_{i}\right)}{d r^{2}} & \ldots & \frac{d^{2} E I_{x y}\left(r_{i}\right)}{d r^{2}} \frac{d^{2} B_{N}\left(r_{i}\right)}{d r^{2}}
\end{array}\right]\left[\begin{array}{llll}
\bar{b}_{k 1} & \bar{b}_{k 2} & \ldots & \bar{b}_{k N}
\end{array}\right]^{T} \\
& +\left[\begin{array}{lllll}
2 \frac{d E I_{x y}\left(r_{i}\right)}{d r} \frac{d^{3} B_{1}\left(r_{i}\right)}{d r^{3}} & 2 \frac{d E I_{x y}\left(r_{i}\right)}{d r} \frac{d^{3} B_{2}\left(r_{i}\right)}{d r^{3}} & \cdots & 2 \frac{d E I_{x y}\left(r_{i}\right)}{d r} \frac{d^{3} B_{N}\left(r_{i}\right)}{d r^{3}}
\end{array}\right]\left[\begin{array}{llll}
\bar{b}_{k 1} & \bar{b}_{k 2} & \cdots & \bar{b}_{k N}
\end{array}\right]^{T}
\end{aligned}
$$


$+\left[\begin{array}{llll}E I_{x y}\left(r_{i}\right) \frac{d^{4} B_{1}\left(r_{i}\right)}{d r^{4}} & E I_{x y}\left(r_{i}\right) \frac{d^{4} B_{2}\left(r_{i}\right)}{d r^{4}} & \cdots & E I_{x y}\left(r_{i}\right) \frac{d^{4} B_{N}\left(r_{i}\right)}{d r^{4}}\end{array}\right]\left[\begin{array}{llll}\bar{b}_{k 1} & \bar{b}_{k 2} & \cdots & \bar{b}_{k N}\end{array}\right]^{T}$

$-\left[\begin{array}{lllll}\rho A\left(r_{i}\right) \Omega^{2}(\sin \theta)^{2} B_{1}\left(r_{i}\right) & \rho A\left(r_{i}\right) \Omega^{2}(\sin \theta)^{2} B_{2}\left(r_{i}\right) & \cdots & \rho A\left(r_{i}\right) \Omega^{2}(\sin \theta)^{2} B_{N}\left(r_{i}\right)\end{array}\right]\left[\begin{array}{llll}b_{k 1} & b_{k 2} & \cdots & b_{k N}\end{array}\right]^{T}$

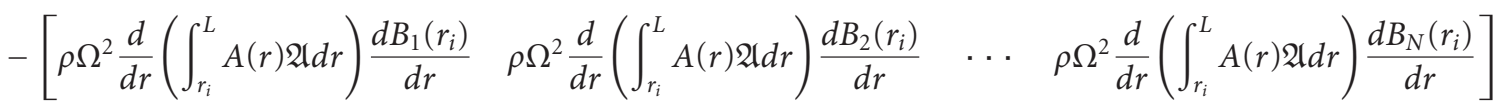

$\times\left[\begin{array}{llll}b_{k 1} & b_{k 2} & \cdots & b_{k N}\end{array}\right]^{T}$

$-\left[\begin{array}{llll}\rho \Omega^{2} \int_{r_{i}}^{L} A(r) \mathfrak{A} d r \frac{d^{2} B_{1}\left(r_{i}\right)}{d r^{2}} & \rho \Omega^{2} \int_{r_{i}}^{L} A(r) \mathfrak{A} d r \frac{d^{2} B_{2}\left(r_{i}\right)}{d r^{2}} & \cdots & \rho \Omega^{2} \int_{r_{i}}^{L} A(r) \mathfrak{A} d r \frac{d^{2} B_{N}\left(r_{i}\right)}{d r^{2}}\end{array}\right]$

$\times\left[\begin{array}{llll}b_{k 1} & b_{k 2} & \cdots & b_{k N}\end{array}\right]^{T}=[0]$ for $i=1,2, \ldots, N, k=1,2,3$,

$\left[\begin{array}{lllll}\lambda^{2} \rho A\left(r_{i}\right) B_{1}\left(r_{i}\right) & \lambda^{2} \rho A\left(r_{i}\right) B_{2}\left(r_{i}\right) & \cdots & \lambda^{2} \rho A\left(r_{i}\right) B_{N}\left(r_{i}\right)\end{array}\right]\left[\begin{array}{lllll}\bar{b}_{k 1} & \bar{b}_{k 2} & \cdots & \bar{b}_{k N}\end{array}\right]^{T}$

$+\left[\begin{array}{lllll}\lambda C_{0} B_{1}\left(r_{i}\right) & \lambda C_{0} B_{2}\left(r_{i}\right) & \cdots & \lambda C_{0} B_{N}\left(r_{i}\right)\end{array}\right]\left[\begin{array}{llll}\bar{b}_{k 1} & \bar{b}_{k 2} & \cdots & \bar{b}_{k N}\end{array}\right]^{T}$

$+\left[\begin{array}{lllll}\lambda C_{1} \frac{d^{2} E I_{x x}\left(r_{i}\right)}{d r^{2}} \frac{d^{2} B_{1}\left(r_{i}\right)}{d r^{2}} & \lambda C_{1} \frac{d^{2} E I_{x x}\left(r_{i}\right)}{d r^{2}} \frac{d^{2} B_{2}\left(r_{i}\right)}{d r^{2}} & \cdots & \lambda C_{1} \frac{d^{2} E I_{x x}\left(r_{i}\right)}{d r^{2}} \frac{d^{2} B_{N}\left(r_{i}\right)}{d r^{2}}\end{array}\right]\left[\begin{array}{llll}\bar{b}_{k 1} & \bar{b}_{k 2} & \cdots & \bar{b}_{k N}\end{array}\right]^{T}$

$+\left[2 \lambda C_{1} \frac{d E I_{x x}\left(r_{i}\right)}{d r} \frac{d^{3} B_{1}\left(r_{i}\right)}{d r^{3}} \quad 2 \lambda C_{1} \frac{d E I_{x x}\left(r_{i}\right)}{d r} \frac{d^{3} B_{2}\left(r_{i}\right)}{d r^{3}} \quad \cdots \quad 2 \lambda C_{1} \frac{d E I_{x x}\left(r_{i}\right)}{d r} \frac{d^{3} B_{N}\left(r_{i}\right)}{d r^{3}}\right]\left[\begin{array}{llll}\bar{b}_{k 1} & \bar{b}_{k 2} & \cdots & \bar{b}_{k N}\end{array}\right]^{T}$

$+\left[\begin{array}{lllll}\lambda C_{1} E I_{x x}\left(r_{i}\right) \frac{d^{4} B_{1}\left(r_{i}\right)}{d r^{4}} & \lambda C_{1} E I_{x x}\left(r_{i}\right) \frac{d^{4} B_{2}\left(r_{i}\right)}{d r^{4}} & \cdots & \lambda C_{1} E I_{x x}\left(r_{i}\right) \frac{d^{4} B_{N}\left(r_{i}\right)}{d r^{4}}\end{array}\right]\left[\begin{array}{llll}\bar{b}_{k 1} & \bar{b}_{k 2} & \cdots & \bar{b}_{k N}\end{array}\right]^{T}$

$+\left[\begin{array}{lllll}\lambda C_{1} \frac{d^{2} E I_{x y}\left(r_{i}\right)}{d r^{2}} \frac{d^{2} B_{1}\left(r_{i}\right)}{d r^{2}} & \lambda C_{1} \frac{d^{2} E I_{x y}\left(r_{i}\right)}{d r^{2}} \frac{d^{2} B_{2}\left(r_{i}\right)}{d r^{2}} & \cdots & \lambda C_{1} \frac{d^{2} E I_{x y}\left(r_{i}\right)}{d r^{2}} \frac{d^{2} B_{N}\left(r_{i}\right)}{d r^{2}}\end{array}\right]\left[\begin{array}{llll}b_{k 1} & b_{k 2} & \cdots & b_{k N}\end{array}\right]^{T}$

$+\left[2 \lambda C_{1} \frac{d E I_{x y}\left(r_{i}\right)}{d r} \frac{d^{3} B_{1}\left(r_{i}\right)}{d r^{3}} \quad 2 \lambda C_{1} \frac{d E I_{x y}\left(r_{i}\right)}{d r} \frac{d^{3} B_{2}\left(r_{i}\right)}{d r^{3}} \quad \cdots \quad 2 \lambda C_{1} \frac{d E I_{x y}\left(r_{i}\right)}{d r} \frac{d^{3} B_{N}\left(r_{i}\right)}{d r^{3}}\right]\left[\begin{array}{llll}b_{k 1} & b_{k 2} & \cdots & b_{k N}\end{array}\right]^{T}$

$+\left[\begin{array}{lllll}\lambda C_{1} E I_{x y}\left(r_{i}\right) \frac{d^{4} B_{1}\left(r_{i}\right)}{d r^{4}} & \lambda C_{1} E I_{x y}\left(r_{i}\right) \frac{d^{4} B_{2}\left(r_{i}\right)}{d r^{4}} & \cdots & \lambda C_{1} E I_{x y}\left(r_{i}\right) \frac{d^{4} B_{N}\left(r_{i}\right)}{d r^{4}}\end{array}\right]\left[\begin{array}{llll}b_{k 1} & b_{k 2} & \cdots & b_{k N}\end{array}\right]^{T}$

$+\left[\begin{array}{lllll}\frac{d^{2} E I_{x x}\left(r_{i}\right)}{d r^{2}} \frac{d^{2} B_{1}\left(r_{i}\right)}{d r^{2}} & \frac{d^{2} E I_{x x}\left(r_{i}\right)}{d r^{2}} \frac{d^{2} B_{2}\left(r_{i}\right)}{d r^{2}} & \ldots & \frac{d^{2} E I_{x x}\left(r_{i}\right)}{d r^{2}} \frac{d^{2} B_{N}\left(r_{i}\right)}{d r^{2}}\end{array}\right]\left[\begin{array}{llll}\bar{b}_{k 1} & \bar{b}_{k 2} & \ldots & \bar{b}_{k N}\end{array}\right]^{T}$

$+\left[\begin{array}{lllll}2 \frac{d E I_{x x}\left(r_{i}\right)}{d r} \frac{d^{3} B_{1}\left(r_{i}\right)}{d r^{3}} & 2 \frac{d E I_{x x}\left(r_{i}\right)}{d r} \frac{d^{3} B_{2}\left(r_{i}\right)}{d r^{3}} & \cdots & 2 \frac{d E I_{x x}\left(r_{i}\right)}{d r} \frac{d^{3} B_{N}\left(r_{i}\right)}{d r^{3}}\end{array}\right]\left[\begin{array}{lllll}\bar{b}_{k 1} & \bar{b}_{k 2} & \cdots & \bar{b}_{k N}\end{array}\right]^{T}$

$+\left[\begin{array}{llll}E I_{x x}\left(r_{i}\right) \frac{d^{4} B_{1}\left(r_{i}\right)}{d r^{4}} & E I_{x x}\left(r_{i}\right) \frac{d^{4} B_{2}\left(r_{i}\right)}{d r^{4}} & \cdots & E I_{x x}\left(r_{i}\right) \frac{d^{4} B_{N}\left(r_{i}\right)}{d r^{4}}\end{array}\right]\left[\begin{array}{llll}\bar{b}_{k 1} & \bar{b}_{k 2} & \cdots & \bar{b}_{k N}\end{array}\right]^{T}$

$+\left[\begin{array}{llll}\frac{d^{2} E I_{x y}\left(r_{i}\right)}{d r^{2}} \frac{d^{2} B_{1}\left(r_{i}\right)}{d r^{2}} & \frac{d^{2} E I_{x y}\left(r_{i}\right)}{d r^{2}} \frac{d^{2} B_{2}\left(r_{i}\right)}{d r^{2}} & \cdots & \frac{d^{2} E I_{x y}\left(r_{i}\right)}{d r^{2}} \frac{d^{2} B_{N}\left(r_{i}\right)}{d r^{2}}\end{array}\right]\left[\begin{array}{lllll}b_{k 1} & b_{k 2} & \cdots & b_{k N}\end{array}\right]^{T}$

$+\left[\begin{array}{lllll}2 \frac{d E I_{x y}\left(r_{i}\right)}{d r} \frac{d^{3} B_{1}\left(r_{i}\right)}{d r^{3}} & 2 \frac{d E I_{x y}\left(r_{i}\right)}{d r} \frac{d^{3} B_{2}\left(r_{i}\right)}{d r^{3}} & \cdots & 2 \frac{d E I_{x y}\left(r_{i}\right)}{d r} \frac{d^{3} B_{N}\left(r_{i}\right)}{d r^{3}}\end{array}\right]\left[\begin{array}{lllll}b_{k 1} & b_{k 2} & \cdots & b_{k N}\end{array}\right]^{T}$

$+\left[\begin{array}{llll}E I_{x y}\left(r_{i}\right) \frac{d^{4} B_{1}\left(r_{i}\right)}{d r^{4}} & E I_{x y}\left(r_{i}\right) \frac{d^{4} B_{2}\left(r_{i}\right)}{d r^{4}} & \cdots & E I_{x y}\left(r_{i}\right) \frac{d^{4} B_{N}\left(r_{i}\right)}{d r^{4}}\end{array}\right]\left[\begin{array}{llll}b_{k 1} & b_{k 2} & \cdots & b_{k N}\end{array}\right]^{T}$

$-\left[\begin{array}{lllll}\rho A\left(r_{i}\right) \Omega^{2}(\cos \theta)^{2} B_{1}\left(r_{i}\right) & \rho A\left(r_{i}\right) \Omega^{2}(\cos \theta)^{2} B_{2}\left(r_{i}\right) & \cdots & \rho A\left(r_{i}\right) \Omega^{2}(\cos \theta)^{2} B_{N}\left(r_{i}\right)\end{array}\right]\left[\begin{array}{llll}\bar{b}_{k 1} & \bar{b}_{k 2} & \cdots & \bar{b}_{k N}\end{array}\right]^{T}$ 


$$
\begin{aligned}
& -\left[\begin{array}{llll}
\rho \Omega^{2} \frac{d}{d r}\left(\int_{r_{i}}^{L} A(r) \mathfrak{A} d r\right) \frac{d B_{1}\left(r_{i}\right)}{d r} & \rho \Omega^{2} \frac{d}{d r}\left(\int_{r_{i}}^{L} A(r) \mathfrak{A} d r\right) \frac{d B_{2}\left(r_{i}\right)}{d r} & \cdots & \rho \Omega^{2} \frac{d}{d r}\left(\int_{r_{i}}^{L} A(r) \mathfrak{A} d r\right) \frac{d B_{N}\left(r_{i}\right)}{d r}
\end{array}\right] \\
& \times\left[\begin{array}{llll}
\bar{b}_{k 1} & \bar{b}_{k 2} & \cdots & \bar{b}_{k N}
\end{array}\right]^{T} \\
& -\left[\begin{array}{lllll}
\rho \Omega^{2} \int_{r_{i}}^{L} A(r) \mathfrak{A} d r \frac{d^{2} B_{1}\left(r_{i}\right)}{d r^{2}} & \rho \Omega^{2} \int_{r_{i}}^{L} A(r) \mathfrak{A} d r \frac{d^{2} B_{2}\left(r_{i}\right)}{d r^{2}} & \cdots & \rho \Omega^{2} \int_{r_{i}}^{L} A(r) \mathfrak{A} d r \frac{d^{2} B_{N}\left(r_{i}\right)}{d r^{2}}
\end{array}\right] \\
& \times\left[\begin{array}{llll}
\bar{b}_{k 1} & \bar{b}_{k 2} & \cdots & \bar{b}_{k N}
\end{array}\right]^{T}=\left[\begin{array}{lll}
0 & \text { for } i=1,2, \ldots, N, k=1,2,3 .
\end{array}\right.
\end{aligned}
$$

where $\mathfrak{A}$ denotes $\left(r+\bar{r}_{0}\right)$.

According to the radial basis function approach, the boundary conditions in (14) have the following discrete forms:

$$
\begin{aligned}
& {\left[\begin{array}{lllll}
B_{1}\left(r_{1}\right) & B_{2}\left(r_{1}\right) & \cdots & B_{N}\left(r_{1}\right)
\end{array}\right]\left[\begin{array}{llll}
b_{k 1} & b_{k 2} & \cdots & b_{k N}
\end{array}\right]^{T}=[0] \text { for } k=1,2,3,} \\
& {\left[\begin{array}{llll}
\frac{d B_{1}\left(r_{1}\right)}{d r} & \frac{d B_{2}\left(r_{1}\right)}{d r} & \ldots & \frac{d B_{N}\left(r_{1}\right)}{d r}
\end{array}\right]\left[\begin{array}{llll}
b_{k 1} & b_{k 2} & \ldots & b_{k N}
\end{array}\right]^{T}=\left[\begin{array}{l}
0
\end{array}\right] \quad \text { for } k=1,2,3,} \\
& {\left[\begin{array}{llll}
E I_{y y}\left(r_{N}\right) \frac{d^{2} B_{1}\left(r_{N}\right)}{d r^{2}} & E I_{y y}\left(r_{N}\right) \frac{d^{2} B_{2}\left(r_{N}\right)}{d r^{2}} & \cdots & E I_{y y}\left(r_{N}\right) \frac{d^{2} B_{N}\left(r_{N}\right)}{d r^{2}}
\end{array}\right]\left[\begin{array}{llll}
b_{k 1} & b_{k 2} & \cdots & b_{k N}
\end{array}\right]^{T}=[0] \quad \text { for } k=1,2,3 \text {, }} \\
& {\left[\begin{array}{lllll}
\frac{d E I_{y y}\left(r_{N}\right)}{d r} \frac{d^{2} B_{1}\left(r_{N}\right)}{d r^{2}} & \frac{d E I_{y y}\left(r_{N}\right)}{d r} \frac{d^{2} B_{2}\left(r_{N}\right)}{d r^{2}} & \cdots & \frac{d E I_{y y}\left(r_{N}\right)}{d r} \frac{d^{2} B_{N}\left(r_{N}\right)}{d r^{2}}
\end{array}\right]\left[\begin{array}{llll}
b_{k 1} & b_{k 2} & \cdots & b_{k N}
\end{array}\right]^{T}} \\
& +\left[\begin{array}{llll}
E I_{y y}\left(r_{N}\right) \frac{d^{3} B_{1}\left(r_{N}\right)}{d r^{3}} & E I_{y y}\left(r_{N}\right) \frac{d^{3} B_{2}\left(r_{N}\right)}{d r^{3}} & \cdots & E I_{y y}\left(r_{N}\right) \frac{d^{3} B_{N}\left(r_{N}\right)}{d r^{3}}
\end{array}\right] \\
& \times\left[\begin{array}{llll}
b_{k 1} & b_{k 2} & \cdots & b_{k N}
\end{array}\right]^{T}=[0] \quad \text { for } k=1,2,3, \\
& {\left[\begin{array}{llll}
B_{1}\left(r_{1}\right) & B_{2}\left(r_{1}\right) & \cdots & B_{N}\left(r_{1}\right)
\end{array}\right]\left[\begin{array}{llll}
\bar{b}_{k 1} & \bar{b}_{k 2} & \cdots & \bar{b}_{k N}
\end{array}\right]^{T}=[0] \text { for } k=1,2,3,} \\
& {\left[\begin{array}{llll}
\frac{d B_{1}\left(r_{1}\right)}{d r} & \frac{d B_{2}\left(r_{1}\right)}{d r} & \ldots & \frac{d B_{N}\left(r_{1}\right)}{d r}
\end{array}\right]\left[\begin{array}{llll}
\bar{b}_{k 1} & \bar{b}_{k 2} & \ldots & \bar{b}_{k N}
\end{array}\right]^{T}=[0] \quad \text { for } k=1,2,3,} \\
& {\left[\begin{array}{llll}
E I_{x x}\left(r_{N}\right) \frac{d^{2} B_{1}\left(r_{N}\right)}{d r^{2}} & E I_{x x}\left(r_{N}\right) \frac{d^{2} B_{2}\left(r_{N}\right)}{d r^{2}} & \cdots & E I_{x x}\left(r_{N}\right) \frac{d^{2} B_{N}\left(r_{N}\right)}{d r^{2}}
\end{array}\right]\left[\begin{array}{llll}
\bar{b}_{k 1} & \bar{b}_{k 2} & \cdots & \bar{b}_{k N}
\end{array}\right]^{T}=\left[\begin{array}{ll}
0 & \text { for } k=1,2,3,
\end{array}\right.} \\
& {\left[\begin{array}{lllll}
\frac{d E I_{x x}\left(r_{N}\right)}{d r} \frac{d^{2} B_{1}\left(r_{N}\right)}{d r^{2}} & \frac{d E I_{x x}\left(r_{N}\right)}{d r} \frac{d^{2} B_{2}\left(r_{N}\right)}{d r^{2}} & \ldots & \frac{d E I_{x x}\left(r_{N}\right)}{d r} \frac{d^{2} B_{N}\left(r_{N}\right)}{d r^{2}}
\end{array}\right]\left[\begin{array}{llll}
\bar{b}_{k 1} & \bar{b}_{k 2} & \ldots & \bar{b}_{k N}
\end{array}\right]^{T}} \\
& +\left[\begin{array}{llll}
E I_{x x}\left(r_{N}\right) \frac{d^{3} B_{1}\left(r_{N}\right)}{d r^{3}} & E I_{x x}\left(r_{N}\right) \frac{d^{3} B_{2}\left(r_{N}\right)}{d r^{3}} & \cdots & E I_{x x}\left(r_{N}\right) \frac{d^{3} B_{N}\left(r_{N}\right)}{d r^{3}}
\end{array}\right] \\
& \times\left[\begin{array}{llll}
\bar{b}_{k 1} & \bar{b}_{k 2} & \cdots & \bar{b}_{k N}
\end{array}\right]^{T}=[0] \quad \text { for } k=1,2,3 .
\end{aligned}
$$

\section{Results}

Figure 1 illustrates the effects of various rotational speeds on calculated frequencies for a wind turbine blade. Computational results suggest that the first frequency of a wind turbine blade is strongly dependent on blade shaft speed. The first frequency of a wind turbine blade increases as rotational speed increases. Numerical results obtained by this study suggest that rotational speed does not significantly affect the second, third, and fourth frequencies of a wind blade. Figure 2 lists the frequencies of a wind turbine blade at different pitch angles. Numerical results demonstrate that pitch angle does not significantly affect the first, second, third, and fourth frequencies of a wind turbine blade. 


\section{Concluding Remarks}

The radial basis function scheme has been extensively adopted to solve various problems in different scientific and engineering fields. This study investigated how pitch angles and rotational speeds affect frequencies of a wind generator blade. The simplicity of this formulation makes it a good candidate for very complex applications.

\section{References}

[1] T. K. Fung, R. L. Scheffler, and J. Stolpe, "Wind energy-a utility perspective," IEEE Transactions on Power Apparatus and Systems, vol. 100, no. 3, pp. 1176-1182, 1981.

[2] G. M. J. Herbert, S. Iniyan, E. Sreevalsan, and S. Rajapandian, "A review of wind energy technologies," Renewable and Sustainable Energy Reviews, vol. 11, no. 6, pp. 1117-1145, 2007.

[3] A. Varol, C. Ilkilic, and Y. Varol, "Increasing the efficiency of wind turbines," Journal of Wind Engineering and Industrial Aerodynamics, vol. 89, no. 9, pp. 809-815, 2001.

[4] R. Spee, S. Bhowmik, and J. H. R. Enslin, "Novel control strategies for variable-speed doubly fed wind power generation systems," Renewable Energy, vol. 6, no. 8, pp. 907-915, 1995.

[5] A. J. Vitale and A. P. Rossi, "Computational method for the design of wind turbine blades," International Journal of Hydrogen Energy, vol. 33, no. 13, pp. 3466-3470, 2008.

[6] Y. D. Song and B. Dhinakaran, "Nonlinear variable speed control of wind turbines," in Proceedings of the IEEE International Conference on Control Applications (CCA '99), pp. 814-819, August 1999.

[7] D. Storti and Y. Aboelnaga, "Bending vibrations of a class of rotating beams with hypergeometric solutions," Journal of Applied Mechanics, vol. 54, no. 2, pp. 311-314, 1987.

[8] J. S. Rao, "Flexural vibration of pretwisted tapered cantilever blades," ASME Journal of Engineering for Industry, vol. 94, no. 1, pp. 343-346, 1972.

[9] J. S. Rao, "Vibrations of rotating, pretwisted and tapered blades," Mechanism and Machine Theory, vol. 12, no. 4, pp. 331-337, 1977.

[10] S. Abrate, "Vibration of non-uniform rods and beams," Journal of Sound and Vibration, vol. 185, no. 4, pp. 703-716, 1995.

[11] D. H. Hodges, Y. Y. Chung, and X. Y. Shang, "Discrete transfer matric method for non-uniform rotating beams," Journal of Sound and Vibration, vol. 169, no. 2, pp. 276-283, 1994.

[12] B. Dawson, "Coupled bending-bending vibrations of pretwisted cantilever blading treated by Rayleigh-Ritz energy method," Journal of Mechanical Engineering Science, vol. 10, pp. 381-386, 1968.

[13] B. Dawson and W. Carneige, "Model curves of pretwisted beams of rectangular cross-section," Journal of Mechanical Engineering Science, vol. 11, pp. 1-13, 1969.

[14] R. S. Gupta and S. S. Rao, "Finite element eigenvalue analysis of tapered and twisted Timoshenko beams," Journal of Sound and Vibration, vol. 56, no. 2, pp. 187-200, 1978.

[15] M. Swaminathan and J. S. Rao, "Vibrations of rotating, pretwisted and tapered blades," Mechanism and Machine Theory, vol. 12, no. 4, pp. 331-337, 1977.

[16] K. B. Subrahmanyam, S. V. Kulkarni, and J. S. Rao, "Coupled bending-bending vibrations of pre-twisted cantilever blading allowing for shear deflection and rotary inertia by the Reissner method," International Journal of Mechanical Sciences, vol. 23, no. 9, pp. 517-530, 1981.

[17] K. B. Subrahmanyam and J. S. Rao, "Coupled bendingbending vibrations of pretwisted tapered cantilever beams treated by the Reissner method," Journal of Sound and Vibration, vol. 82, no. 4, pp. 577-592, 1982.

[18] W. R. Chen and L. M. Keer, "Transverse vibrations of a rotating twisted Timshenko beam under axial loading," ASME Journal of Vibration and Acoustics, vol. 115, pp. 285-294, 1993.

[19] D. Storti and Y. Aboelnaga, "Bending vibrations of a class of rotating beams with hypergeometric solutions," Journal of Applied Mechanics, vol. 54, no. 2, pp. 311-314, 1987.

[20] J. T. Wagner, "Coupling of turbomachine blade vibrations through the rotor," ASME Journal of Engineering for Power, vol. 89, pp. 502-512, 1967.

[21] J. H. Griffin, "Friction damping of resonant stresses in gas turbine engine airfoils," ASME Journal of Engineering for Power, vol. 102, no. 2, pp. 329-333, 1980.

[22] J. H. Griffin and A. Sinha, "The interaction between mistuning and friction in the forced response of bladed disk assemblies," ASME Journal of Engineering for Gas Turbines and Power, vol. 107, no. 1, pp. 107-205, 1985.

[23] A. Sinha and J. H. Griffin, "Effects of static friction on the forced response of frictionally damped turbine blades," ASME Journal of Engineering for Gas Turbines and Power, vol. 106, no. 1, pp. 65-69, 1984.

[24] E. J. Kansa, "Multiquadrics-a scattered data approximation scheme with applications to computational fluid-dynamicsI surface approximations and partial derivative estimates," Computers and Mathematics with Applications, vol. 19, no. 8-9, pp. 127-145, 1990.

[25] E. J. Kansa, "Multiquadrics-a scattered data approximation scheme with applications to computational fluid-dynamics-II solutions to parabolic, hyperbolic and elliptic partial differential equations," Computers and Mathematics with Applications, vol. 19, no. 8-9, pp. 147-161, 1990.

[26] J. G. Wang and G. R. Liu, "A point interpolation meshless method based on radial basis functions," International Journal for Numerical Methods in Engineering, vol. 54, no. 11, pp. 1623-1648, 2002.

[27] L. A. Elfelsoufi, "Buckling, flutter and vibration analyses of beams by integral equation formulations," Computers and Structures, vol. 83, no. 31-32, pp. 2632-2649, 2005.

[28] Y. C. Hon, M. W. Lu, W. M. Xue, and Y. M. Zhu, "Multiquadric method for the numerical solution of a biphasic mixture model," Applied Mathematics and Computation, vol. 88, no. 23, pp. 153-175, 1997.

[29] G. R. Liu, X. Zhao, K. Y. Dai, Z. H. Zhong, G. Y. Li, and X. Han, "Static and free vibration analysis of laminated composite plates using the conforming radial point interpolation method," Composites Science and Technology, vol. 68, no. 2, pp. 354-366, 2008.

[30] K. N. Devi and D. W. Pepper, "A meshless radial basis function method for fluid flow with heat transfer," International Conference on Computational and Experimental Engineering and Sciences, vol. 6, no. 1, pp. 13-18, 2008.

[31] L. Vrankar, G. Turk, and F. Runovc, "Modelling of radionuclide migration through the geosphere with radial basis function method and geostatistics," Journal of the Chinese Institute of Engineers, vol. 27, no. 4, pp. 455-462, 2004. 
[32] Y. Qiao and L. Ernst, "Developing a computational radial basis function architecture for nonlinear scattered color data," in Proceedings of the 22nd International Conference on Digital Printing Technologies Final Program and Proceeding, pp. 374377, Denver, Colo, USA, September 2006.

[33] G. B. Wright, Radial basis function interpolation: numerical and analytical developments, Ph.D. thesis, University of Colorado at Boulder, Denver, Colo, USA, 2003.

[34] M. D. Buhmann, Radial Basis Functions: Theory and Implementations, Cambridge University Press, Cambridge, Mass, USA, 2003. 

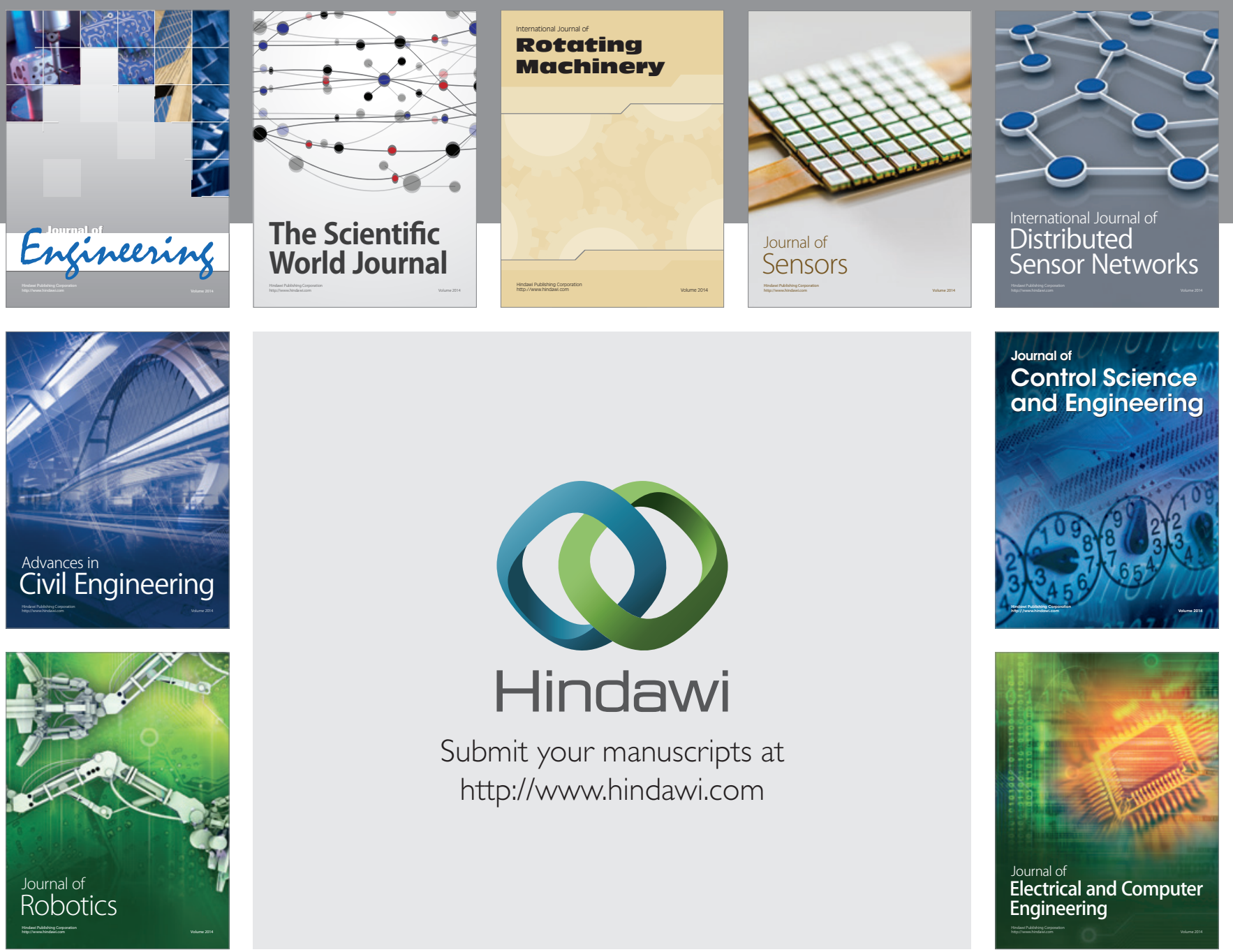

Submit your manuscripts at

http://www.hindawi.com
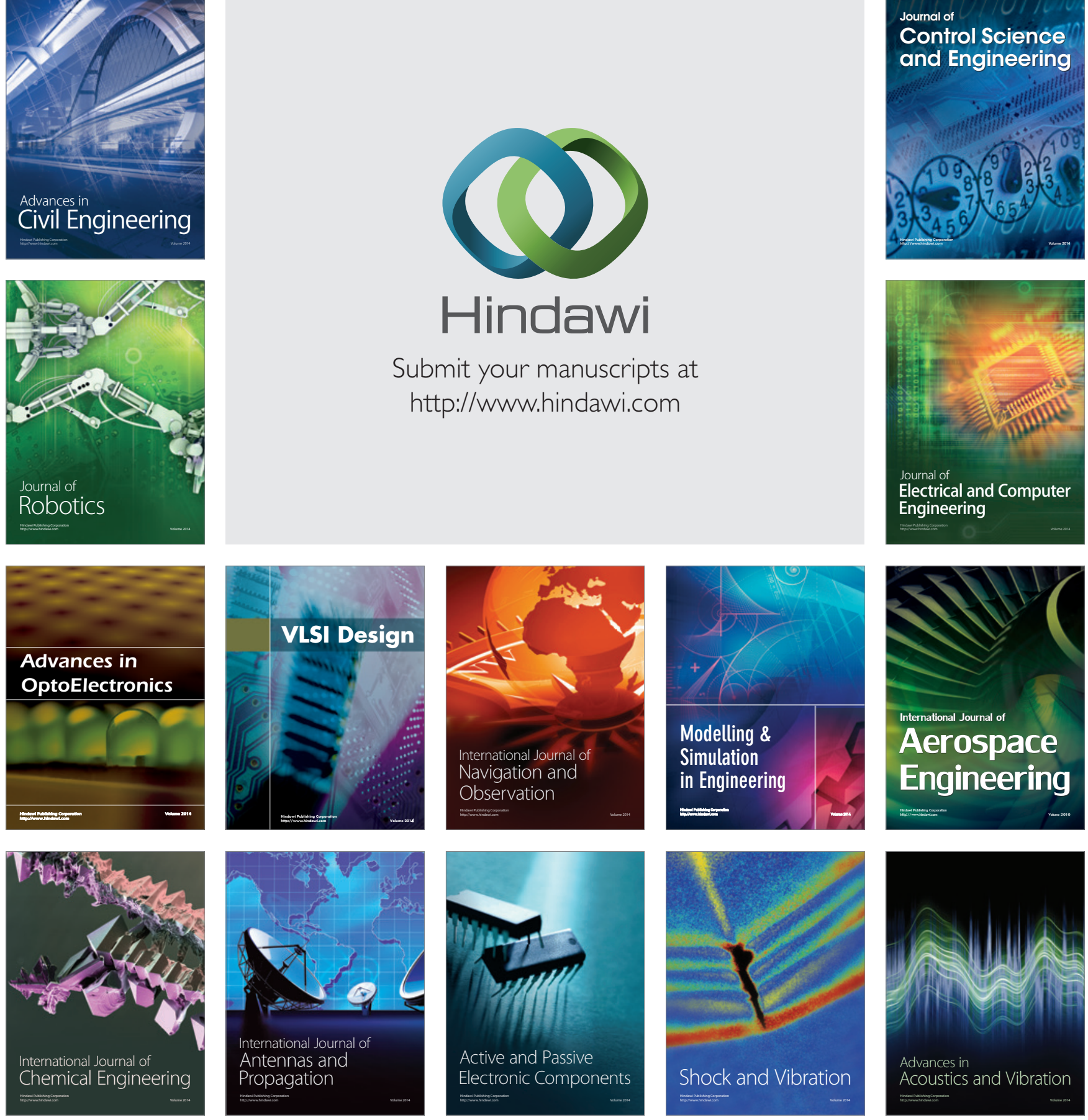